\title{
GPS Tracking App for Police to Track Ambulances Carrying COVID-19 Patients for Ensuring Safe Distancing
}

\author{
Ranajoy Mallik $^{1}$ (C) $\cdot$ Dilip Sing $^{1} \cdot$ Rajib Bandyopadhyay $^{1}$
}

Received: 22 April 2020 / Revised: 21 May 2020 / Accepted: 26 May 2020 / Published online: 8 June 2020

(C) Indian National Academy of Engineering 2020

\begin{abstract}
The outbreak of the SARS-CoV-2 virus is causing loss of lives and property all over the world. There have been more than 2.1 million cases of COVID-19 with a death of more than 1.2 lakh patients worldwide and the numbers are still rising. The virus spreads rapidly by the droplets coming out from the nose and mouth of an infected person (Sandoiu in Why does SARS-CoV-2 spread so easily? Medical news today, 2020 https://www.medicalnewstoday.com/articles/why-does-sars-cov-2-spread-so-easil y). In this situation, proper quarantining and monitoring of the already infected patients are very essential. In cases where patients need to be transferred to different locations by ambulances, monitoring of these ambulances by the traffic police can help to ensure distancing and faster movement of the vehicle inside the city. This paper presents the development of a Real-time Global Positioning System-based tracking app for the ambulances carrying COVID-19 patients which would help traffic police to ensure distancing the patients from the public.
\end{abstract}

Keywords GPS tracking $\cdot$ Android app $\cdot$ Ambulance $\cdot$ Firebase $\cdot$ COVID-19

\section{Introduction}

\section{Inspiration from Existing Technology}

The Global Positioning System (GPS) tracking technology for vehicles has been prevailing in our country since the launch of online Taxi or cab booking system like OLA and Uber. The current technology uses GPS to track their cabs and show their real-time location to the customers who have booked them. The technology has been very successful in exchanging the location information of the drivers and customers to each other which has reduced the burden of calling and enquiring for their location frequently. In this pandemic situation, this technology has the potential to be tweaked to help hospitals and traffic polices monitor the movement of ambulances carrying COVID-19 patients in the city.

\section{Working Principle of the Current Technology}

The current technology uses GPS receivers of the Android mobile phones to gather its location data. The apps are

Ranajoy Mallik

wranajoy@gmail.com

1 Department of Instrumentation and Electronics Engineering, Jadavpur University, Salt Lake Campus, Kolkata 700 106, India primarily developed for cabs and customers to communicate with each other. When a customer wants to hire a cab for himself/herself, he or she uses the app to view the available cabs nearby and then books a cab according to his or her requirements. The location of the cabs and the customers is constantly monitored and their location data are exchanged with each other via an online database. This reduces the hassle of frequently calling or texting in between the cabs and the customers for knowing their locations.

\section{Tweaking of the Technology to Address COVID-19 Pandemic}

We have used this idea as a basis for developing an app which would help hospitals and traffic police to track ambulances carrying COVID-19 patients. COVID-19 is a very contagious disease which spreads very easily (Sandoiu 2020). In the up-coming days, when lockdown will eventually be relaxed, cars and buses will start to operate in the cities. In cities like Bengaluru and Mumbai which have a very high population density, traffic congestion is inevitable. The TomTom traffic index of 2019 declared Bengaluru as the most traffic congested city of the world followed by Mumbai in the 4th place (TomTom Traffic index 2019). During a traffic congestion, transporting a COVID-19 patient 
between two places fast could be a challenge. The location information of an ambulance carrying COVID-19 patients can be useful for the traffic police to isolate the ambulance from the congested area and guide them to the clear roads which would help in faster relocation of the patients.

The ambulances used for COVID-19 emergencies may have traces of the virus even after thorough sanitization. And while carrying the patients, there is a high chance that the exterior surfaces of the ambulance may carry virus. The New England Journal of Medicine claimed that the virus can survive on plastic, stainless steel and glass for more than 2-3 days (van Doremalen et al. 2020). They have also claimed that the virus could survive in droplets for up to $3 \mathrm{~h}$ after being coughed out into the air. This shows that surfaces like door handles and glass panels of the ambulances and even the surrounding air around the ambulances may happen to be contaminated by the virus (BBC 2020). In case of an unlikely event where an ambulance somehow failed to isolate the virus, the ambulance could become an accessory for the spread of SARS-CoV-2 virus to the public. Distancing other vehicles and pedestrian from proximity of the ambulances is crucial. When these ambulances travel around the city, the location of these ambulances may be conveyed to the traffic police, so that they can ensure distancing of these ambulances from the public by clearing the traffic signals on the way of the ambulances and blocking the way of other vehicles and public towards these ambulances. This can be an added safety measure to fight the spread of the SARSCoV-2 virus.

To achieve these purposes, we have developed an android app which can be installed in both the mobile phones of the traffic polices and the drivers of the ambulances. This app would to provide real-time location data of the ambulances to the traffic police directly.

\section{Proposed Work}

\section{Methodology of the Tracking App}

The app provides real-time location tracking of ambulances carrying COVID-19 patients. It uses the in-built Global Positioning System (GPS) of the mobiles to pinpoint the location of the device and upload the location coordinates to a real-time database. The app uses many APIs and services from Google for achieving this purpose. The app is equipped with authentication features like email and password sign-in, Google sign-in, Facebook sign-in and many more to prevent access to unauthorized personnel (Firebase Authentication 2020). The app uses a real-time database called Firebase from Google which is a cloud-hosted NoSQL database that lets us store and sync data between users in real time (Firebase Database 2020). The process of location data exchange in between the ambulance and traffic police is shown in Fig. 1. A screenshot of the data base is given in Fig. 2 where under the user child, the registered ambulance drivers and police are shown, and under the "driversAvailable" child, the drivers who are currently on duty are shown. Another child "policeAvailable" gets created, when a police $\operatorname{logs}$ in the app. There are different user interfaces with specific functionalities for ambulance and traffic police separately (Fig. 3).

The app uses Geofire which is an open-source JavaScript library that allows user to store and query a set of items based on their geographic location (Geofire 2020). The location of the ambulance is gathered by Geofire and is uploaded to the firebase database as soon as the ambulance logs in the app. The location data get updated every second in the database. When a traffic police logs in, the Geofire creates a circle about the position of the police with a predefined radius and searches for all the logged-in ambulances within that radius as shown in Fig. 4. After obtaining the locations
Fig. 1 Exchange of location data using firebase

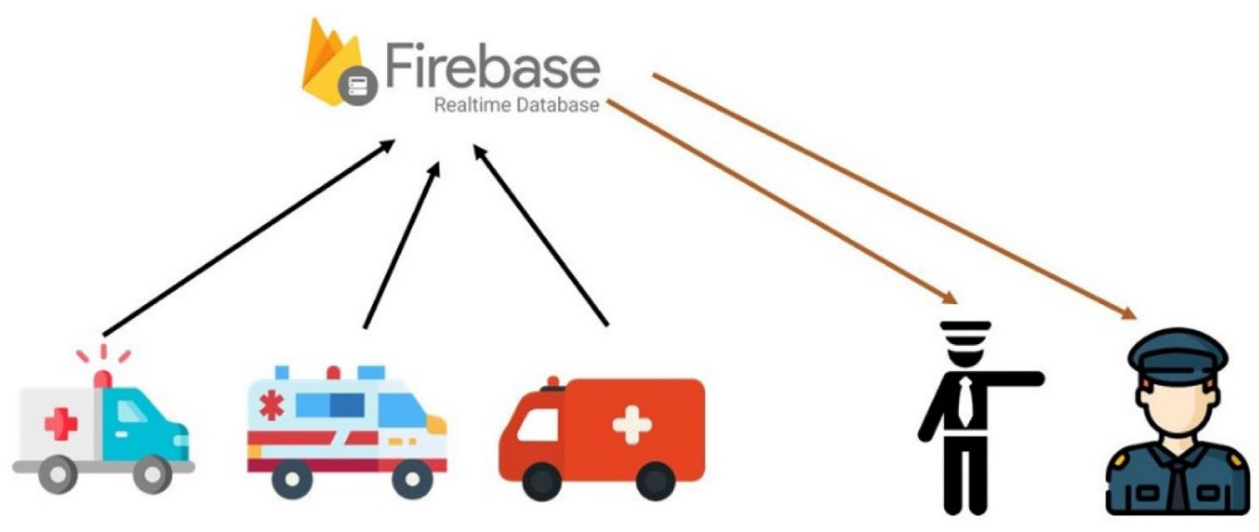


Fig. 2 Firebase real-time database showing the drivers and police c) https://myambulance-965a0.firebaseio.com

$\oplus \ominus$ :

\section{MyAmbulance}

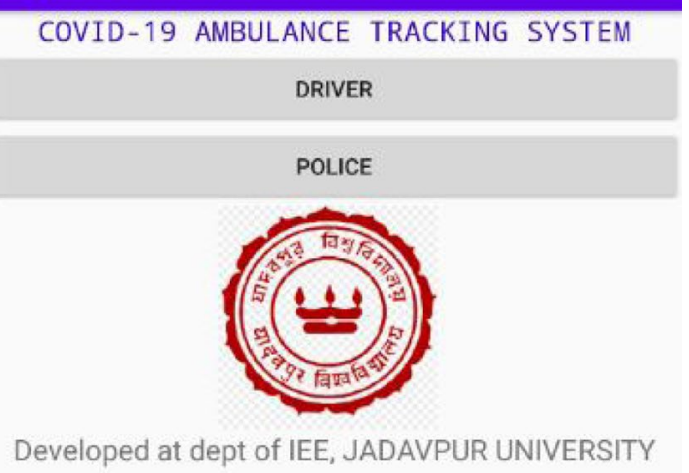

Developed at dept of IEE, JADAVPUR UNIVERSITY

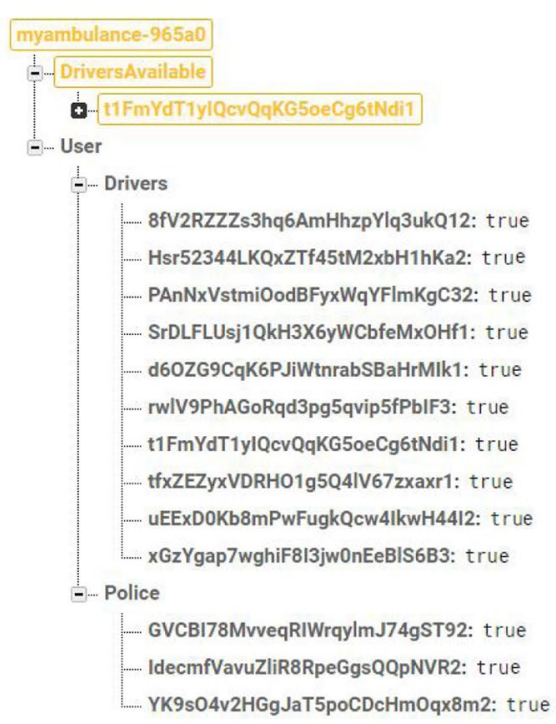

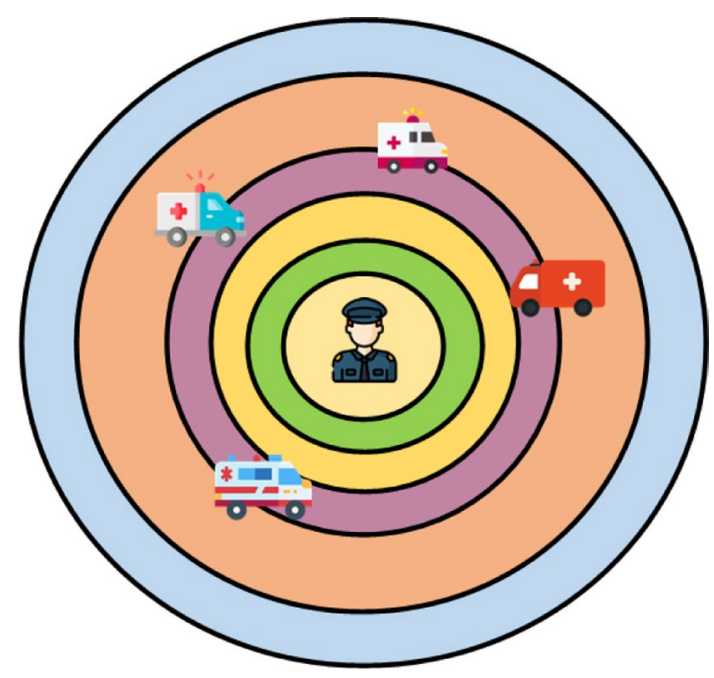

Fig. 4 Tracking of ambulances by Geoquery

can be tracked simultaneously in real time and their position can be shown on the map.

\section{Timeline and Resources for Implementation of the Technology}

The app works with the help of resources that are already available in all android mobile phones. The Android application package file (APK file) can be distributed via emails to all the traffic police headquarters and hospitals. The application can be installed directly in respective mobile phones by the authorized users. As the app is already developed and is
Fig. 3 Different activity screen for Police and ambulance with different functionalities

of all the nearby ambulances, the locations are shown on the maps in real time as shown in Figs. 5 and 6. The app shows the location of the ambulances by the help of Google Maps API and is accurate to about four meters. Many ambulances 


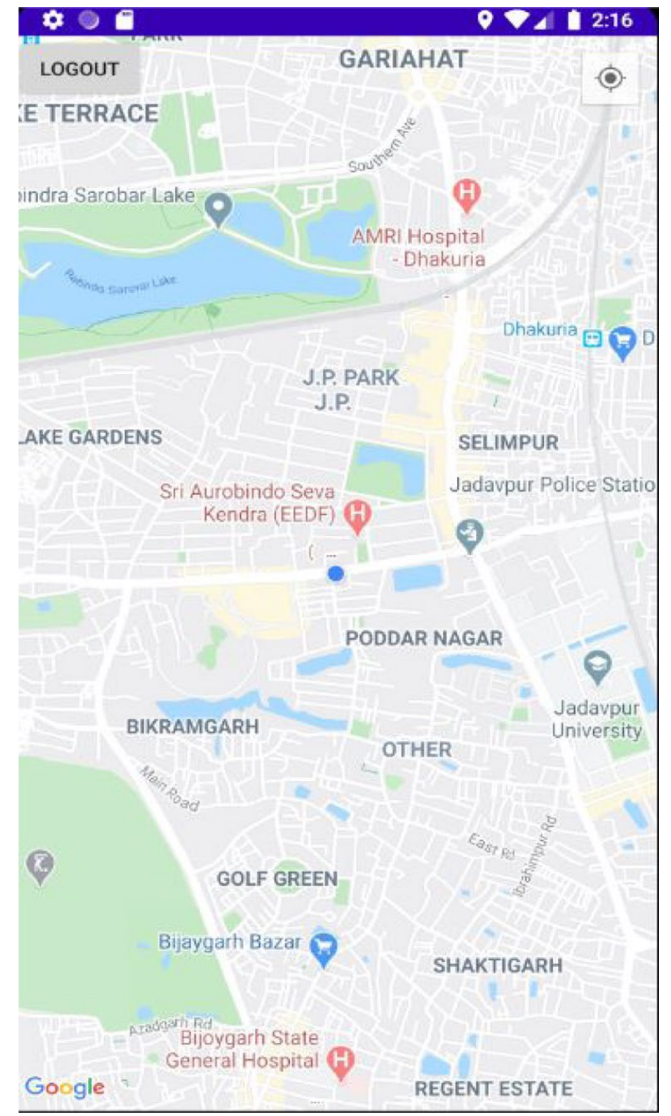

Fig. 5 Ambulance screen showing its own location

being tested in different devices, the implementation of the app can be done at any time by the concerned authorities.

\section{Results and Conclusion}

The app provides an easy and accurate tracking of the ambulances and can, therefore, help in the isolation of infected patients. There are also provisions for assisting the drivers of the ambulances like finding and contacting the nearest traffic police for help. Many more useful and safety features can be incorporated in the app in the future. The app is written in JAVA and is created using Android Studio. It has been successfully tested in many Android devices (Table 1).

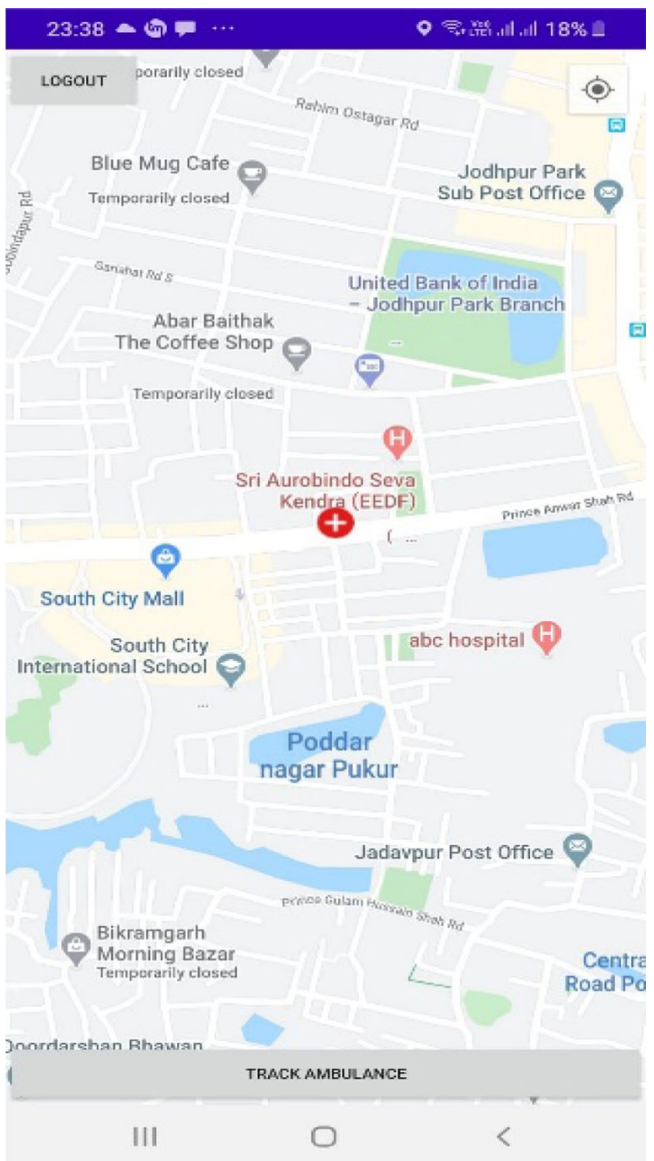

Fig. 6 Traffic police screen showing the location of the ambulance

In the development stage, the billing plan for the database used here is the firebase spark plan which is the free plan provided by Firebase. This plan has few limitations like 200,000 simultaneous connection to the database and the size of data downloaded from the database at a single location should be less than $256 \mathrm{MB}$ for each read operation. These usage limits can be resolved by choosing a different paid plan from Firebase according to requirement.

In the future, a network of apps likes these and combining them with IOT can help people to receive health care services with more ease and faster response. 
Table 1 List of tracked ambulances and their tracking police

\begin{tabular}{|c|c|c|c|c|c|}
\hline $\begin{array}{l}\text { Ambulance/ } \\
\text { police }\end{array}$ & Source & Destination & Tracking police & $\begin{array}{l}\text { Ride time } \\
\text { (min) }\end{array}$ & Remarks \\
\hline A-1 & Gariahat & Park circus & $\mathrm{P}-1, \mathrm{P}-2$ & 25 & P-1 and P-2 tracked all the ambulances simultaneously \\
\hline A-2 & Gariahat & Naktala & P-1, P-2 & 35 & \\
\hline A-3 & Dhakuria & Saltlake & P-1, P-2 & 50 & \\
\hline P-1 & - & Saltlake & - & - & \\
\hline P-2 & - & Dhakuria & - & - & \\
\hline
\end{tabular}

Author Contributions All the authors have contributed in their respective parts.

Funding Not Applicable.

Availability of Data and Material Data and material available.

\section{Compliance with Ethical Standards}

Conflict of Interest There is no conflict of interest.

Code Availability The Java code of the app is available.

\section{References}

van Doremalen N, Bushmaker T, Morris DH, Holbrook MG et al (2020) Aerosol and surface stability of SARS-CoV-2 as compared with SARS-CoV-1. N Engl J Med 382:1564-1567
BBC (2020) Covid-19: how long does the coronavirus last on surfaces?. https://www.bbc.com/future/article/20200317-covid -19-how-long-does-the-coronavirus-last-on-surfaces

Firebase Authentication (2020). https://firebase.google.com/docs/auth Firebase Database (2020). https://firebase.google.com/docs/database

Geofire (2019). https://github.com/firebase/geofire-android

Sandoiu A (2020) Why does SARS-CoV-2 spread so easily? Medical news today. https://www.medicalnewstoday.com/articles/whydoes-sars-cov-2-spread-so-easily

TomTom Traffic index (2019). https://www.tomtom.com/en_gb/traff ic-index/ranking/

Publisher's Note Springer Nature remains neutral with regard to jurisdictional claims in published maps and institutional affiliations. 